\title{
Load Dispatch Optimization using Dynamic Rating and Optimal Lifetime Utilization of Transformers
}

Viafora, Nicola; Kazmi, Syed Hamza Hasan; Olesen, Thomas Herskind; Sørensen, Troels Stybe; Holbøll, Joachim

\section{Published in:}

Proceedings of IEEE PES Powertech 2019

Link to article, DOI:

10.1109/PTC.2019.8811002

Publication date:

2019

Document Version

Peer reviewed version

Link back to DTU Orbit

Citation (APA):

Viafora, N., Kazmi, S. H. H., Olesen, T. H., Sørensen, T. S., \& Holbøll, J. (2019). Load Dispatch Optimization using Dynamic Rating and Optimal Lifetime Utilization of Transformers. In Proceedings of IEEE PES Powertech 2019 [Paper ID 756 ] IEEE. https://doi.org/10.1109/PTC.2019.8811002

\section{General rights}

Copyright and moral rights for the publications made accessible in the public portal are retained by the authors and/or other copyright owners and it is a condition of accessing publications that users recognise and abide by the legal requirements associated with these rights.

- Users may download and print one copy of any publication from the public portal for the purpose of private study or research.

- You may not further distribute the material or use it for any profit-making activity or commercial gain

- You may freely distribute the URL identifying the publication in the public portal 


\title{
Load Dispatch Optimization using Dynamic Rating and Optimal Lifetime Utilization of Transformers
}

\author{
Nicola Viafora*, Syed Hamza H. Kazmi ${ }^{\dagger}$, Thomas H. Olesen ${ }^{\dagger}$, Troels S. Sørensen ${ }^{\dagger}$ and Joachim Holbøll* \\ *Dept. of Electrical Engineering, Technical University of Denmark, Lyngby, Denmark, Email: nicovia@elektro.dtu.dk \\ ${ }^{\dagger}$ Transformer \& HV Components Dept., Ørsted Offshore Wind A/S, Gentofte, Denmark, Email: syeka@orsted.dk
}

\begin{abstract}
Power transformers are critical power system components that are generally loaded conservatively, resulting in marginal utilization of their designed lifetime. Dynamic Transformer Rating (DTR) allows increased utilization of this asset by limiting its Hot Spot Temperature (HST) instead of the per unit load, thereby increasing available network capacity. However, residual lifetime would still be unutilized according to current dimensioning criteria and state-of-the-art lifetime aging models. Therefore, this paper proposes a novel methodology for DTR which assesses thermal aging based on HST history and allows optimal lifetime utilization by loading the transformer beyond its continuous HST limit. The proposed methodology is tested in the day-ahead market using a multi-period DCOPF formulation that accounts for power losses while preserving convexity. The IEEE RTS 24-bus network with additional wind generation is used as a test system using actual weather, load and generation data from Denmark for the period 2014-2016. The proposed DTR approach favours the integration of wind power generation and decreases the cost of load dispatch. This novel DTR approach can be beneficial for applications with limited asset lifetime like offshore windfarms or for postponing necessary grid reinforcements for short period of time.
\end{abstract}

Index Terms-dynamic transformer rating, lifetime model, DCOPF, losses, wind power integration

\section{INTRODUCTION}

The integration of renewable-based energy sources, wind power particularly, in the existing grid can be hindered because the thermal overload capability of the network has bottlenecks. The widespread use of power transformers in the transmission and distribution system makes this component critical for power system operation. Dynamic Transformer Rating (DTR) can help resolve these bottlenecks by allowing the transformers to be overloaded based on their thermal state [1].

Loading guides IEEE C57.91 [2] and IEC 60076-7 [3] allow large power transformers to be dynamically rated up to the Hot Spot Temperature (HST) of $160{ }^{\circ} \mathrm{C}$. But the traditional operation philosophy and protection design prevent transformers from being operated beyond HST of $110{ }^{\circ} \mathrm{C}$, which is rarely reached because of favorable ambient conditions. Consequently, transformers are distinctly underutilized and the remaining lifetime by the end of designed period (usually 35-40 years) is significant. This can influence the business case for applications like offshore windfarms, which are traditionally designed to operate for 25 years. Moreover, optimal transformer utilization can help increase the energy and economic turnover and decrease the Cost of Energy (CoE) for such applications. Transmission system operators instead may benefit from increased network capacity in order to face large and rapid penetration of renewable energy sources and postpone required network reinforcements.

This paper builds upon a recent work in [4], where transformer loadability is directly accounted for in a multi-period DC - Optimal Power Flow (DCOPF) algorithm. The novelty of the proposed DTR approach consists in assessing the remaining transformer lifetime using [2] and [3], based on the load and ambient conditions history. Based on this assessment, the optimal dispatch considers not only transformer thermal dynamics, but aging rate and cumulative lifetime utilization as well. This loading approach results in a controlled accelerated aging but without breaching the designed lifetime limit. As a result, the transformer is used more effectively as compared to other DTR approaches suggested in [5] and [6]. The IEEE RTS 24-bus network with additional wind generation [7] is used as a test system based on actual weather, load and generation data from Denmark for the period 2014-2016. The presented case study demonstrates the relevance of the method as a means to improve the utilization of low-cost wind energy while accounting for power losses in the transmission system.

The remaining paper is organized as follows. The DTR models from [2] are discussed and modified for the optimization problem in Section II. Section III elaborates the thermal aging phenomena in transformer and presents the novel DTR approach for optimal lifetime utilization. The optimization problem for day-ahead dispatch is formulated in Section IV. The case study is presented in Section V, while the results are discussed in Section VI. Section VII concludes the paper.

\section{DynAmic TRAnsformer RATING MODEL}

Dynamic loading of transformers can be performed by determining temperatures which are critical for transformer operation: Top-Oil Temperature (TOT) and Hot-Spot Temperature (HST). This estimation is performed by using the ANSI/IEEE Clause 7 model for TOT and HST estimation [2], because of the well-established popularity in the industry and mathematical suitability as compared to other models [3] [8].

\section{A. Estimation of TOT and HST using IEEE Claus 7 Model}

These temperatures are calculated using the non-linear differential equations (1) - (2) which require further simplification to prevent non-convexity of the optimization problem [2]. 


$$
\begin{aligned}
& \tau_{0} \frac{d \vartheta_{\text {top }}}{d t}+\vartheta_{\text {top }}=\vartheta_{\text {amb }}+\vartheta_{\text {or }}\left(\frac{I_{\text {trf }}^{2} R+1}{R+1}\right)^{\nu} \\
& \tau_{h} \frac{d \vartheta_{\mathrm{hst}}}{d t}+\vartheta_{\text {hst }}=\vartheta_{\text {top }}+\vartheta_{\mathrm{hr}} I_{\text {trf }}^{2 \mu}
\end{aligned}
$$

where $\tau_{0}$ and $\tau_{h}$ are the thermal time constants for oil and winding respectively which are expressed in hours; $\vartheta_{\text {amb }}$ is the ambient temperature in ${ }^{\circ} \mathrm{C} ; \vartheta_{\text {top }}$ and $\vartheta_{\text {hst }}$ represent top-oil and hot-spot temperatures respectively in ${ }^{\circ} \mathrm{C} ; I_{\text {trf }}$ is the transformer load current in p.u. with rated load current as base; $R$ is ratio of load losses to no-load losses at rated load; $\vartheta_{\text {or }}$ in ${ }^{\circ} \mathrm{C}$ is the top-oil rise over ambient temperature $\vartheta_{\text {amb }}$ at rated load, while $\vartheta_{\mathrm{hr}}$ in ${ }^{\circ} \mathrm{C}$ is the rated HST rise over TOT for rated load. The empirically derived exponents $\nu$ and $\mu$ represent the impact of transformer cooling mode (ONAN, OFAF etc.) on the change in thermal resistance and oil viscosity. The constants have different values for different cooling modes, which are provided in [2].

\section{B. Linearization of IEEE Models for TOT and HST}

In order to keep the optimization problem convex, some simplifications are made to the TOT and HST models of (1) and (2). Firstly, the selected transformer is assumed to operate continuously at Oil-Directed-Air-Forced (ODAF) mode, allowing both the constants $\nu$ and $\mu$ to be set to 1 . Secondly, hot-spot temperature is modelled in terms of its steady state value, since hourly values are used in the optimization problem. Therefore it is assumed that short term thermal transients would be extinguished within one hour due to the small thermal time constant, as verified by authors of [4]. As opposed to oil time constant $\tau_{0}$, which is in the range of 60 to 90 minutes, winding time constant $\tau_{h}$ is approximately 7-8 minutes. Resulting topoil and hot-spot temperature dynamics are modelled by means of linearized IEEE thermal models shown in (3) and (4), respectively.

$$
\begin{aligned}
& \vartheta_{\text {top }_{t}}=K_{1} I_{\text {trr }_{t}}^{2}+K_{2} \vartheta_{\mathrm{amb}_{t}}+K_{3} \vartheta_{\text {top }_{t-1}}+K_{4} \\
& \vartheta_{\text {hst }_{t}}=\vartheta_{\text {top }_{t}}+\vartheta_{\mathrm{hr}} I_{\text {trf }_{t}}^{2}
\end{aligned}
$$

Top-oil temperature depends on the squared per unit load $I_{\text {trf }}^{2}$, ambient temperature $\vartheta_{\text {amb }}$ and value of top-oil temperature reached in the previous time step. This latter term is responsible of coupling top-oil temperature values in time thus reflecting the importance of considering recent loading history for transformers. Lastly, Coefficients $K$ are constants that solely depend on transformer construction.

\section{Optimal LifETIME EVAlUATION OF TRANSFORMERS}

\section{A. Thermal Aging of Transformers}

The limit for thermal capacity of a transformer is based on the maximum allowable stress on relevant materials. These limits are effectively explored and defined in ANSI/IEEE C57.91 [2] and IEC 60076-7 [3]. The thermal limits for power transformers greater than 100 MVA rating are provided in Table I for different types of dynamic loading beyond nameplate rating. However, the continuous HST limit for
TABLE I

TEMPERATURE LIMITS FOR TRANSFORMERS [2] [3]

\begin{tabular}{cccc}
\hline & $\begin{array}{c}\text { Normal Cyclic } \\
\text { Loading }\end{array}$ & $\begin{array}{c}\text { Emergency Loading } \\
\text { (long-term) }\end{array}$ & $\begin{array}{c}\text { Emergency Loading } \\
(<30 \text { min })\end{array}$ \\
\hline $\begin{array}{c}\text { Hot Spot } \\
\text { Temp. }\end{array}$ & $120{ }^{\circ} \mathrm{C}$ & $140{ }^{\circ} \mathrm{C}$ & $160 / 180^{\circ} \mathrm{C}$ \\
$\begin{array}{c}\text { Top Oil } \\
\text { Temp. }\end{array}$ & $105{ }^{\circ} \mathrm{C}$ & $115{ }^{\circ} \mathrm{C}$ & $115 / 110^{\circ} \mathrm{C}$ \\
\hline
\end{tabular}

designed transformer lifetime is $110{ }^{\circ} \mathrm{C}$ for thermally upgraded paper. This temperature ceiling is scarcely ever reached because of over-dimensioning, protection philosophies and favorable ambient conditions.

The thermal stress is known to be maximum at HST location. The heat transfer from HST serves as catalyst for chemical reactions, which accelerates the aging of insulation paper [1]. The Arrhenius reaction rate theory has been adapted in [2] to calculate the transformer loss of life. The relative aging rate for a transformer, also called aging acceleration factor $\Lambda$, with thermally upgraded insulation paper is given by (5), while the transformer loss of life is given by (6)

$$
\begin{aligned}
& \Lambda(t)=e^{\left(\frac{15000}{110+273}-\frac{15000}{\vartheta_{\text {hst }}(t)+273}\right)} \\
& \lambda(t)=\int_{t_{0}}^{t} \Lambda(\tau) d \tau
\end{aligned}
$$

where $\Lambda$ is unit-less and represents the aging acceleration factor for reference HST of $110{ }^{\circ} \mathrm{C}$ for thermally upgraded insulation paper; $\vartheta_{\text {hst }}(t)$ is the actual hot spot temperature in ${ }^{\circ} \mathrm{C}$ at time $t ; \lambda(t)$ represents the cumulative loss-of-life for time period from $t_{0}$ up to $t$ and in this paper it is expressed in years. Hence the lifetime utilization of transformer is directly dependent on HST. It must be mentioned that the factor $\Lambda$ represents the thermal aging of paper insulation only and the impacts of residual moisture content in paper and oil along with other aging phenomena on transformer lifetime are not assessed in this paper.

\section{B. Dynamic Rating and Improved Lifetime Utilization}

Static Transformer Rating (STR) limits continuous load current to $1 \mathrm{pu}$ for power transformers and cyclic load current to $1.3 \mathrm{pu}$ [2]-[3]. In contrast to this approach, Dynamic Transformer Rating (DTR) allows the transformer to be loaded based on HST instead of the rated capacity and thereby prevents this temperature from violating the limits of Table I [5] - [6]. The methodology for dynamic rating used in this paper additionally evaluates the consumed lifetime $\lambda$ of a transformer and it sets the loadability accordingly.

Referring to Figure 1, it is assumed that until time $t_{0}$ the transformer has continuously operated at HST of $98{ }^{\circ} \mathrm{C}$. The relative aging rate $\Lambda$ is 0.282 , which is represented by the slope of black line in the figure. Consequently, the transformer lossof-life at this point would be $\lambda_{A}=0.282 t_{0}$. The difference between designed loss-of-life $\lambda_{D}$ for HST of $110{ }^{\circ} \mathrm{C}$ and 
(a)

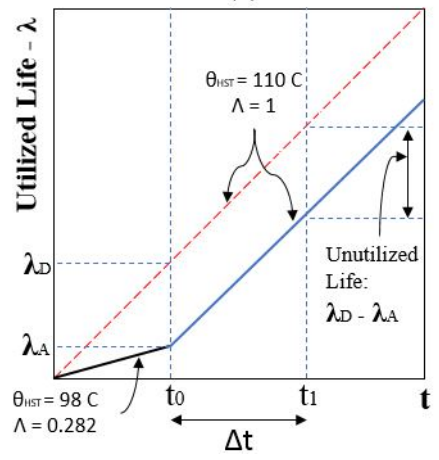

(b)

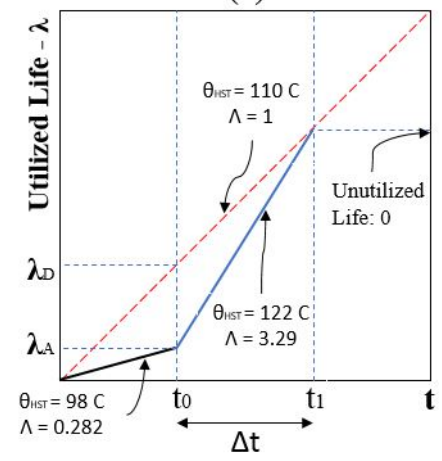

Fig. 1. Methodology for optimal transformer utilization. a: Fixed HST limit of $110 \mathrm{C}$, b: Utilized lifetime $(\lambda)$ dependent HST limit $\left(\vartheta_{\mathrm{hst}, \max }=140 \mathrm{C}\right)$

actual $\lambda$ would continue to increase, if the transformer would keep this loading strategy. DTR can prevent this difference from increasing further by loading the transformer in a way that keeps the HST closer to the design limit of $110{ }^{\circ} \mathrm{C}$, as shown in Figure 1a. But even with this approach, the residual transformer lifetime by the end of design life would be significant. Therefore, the transformer loading strategy proposed in this paper is meant to maximize component's utilization by considering not only temperature dynamics, but aging rate as well. Figure $1 \mathrm{~b}$ illustrates the underlying concept of Enhanced Dynamic Transformer Rating (DTR ${ }^{+}$). For the period between $t_{0}$ and $t_{1}$, the upper HST limit is set to 122 ${ }^{\circ} \mathrm{C}$. As a result of this increased upper temperature limit, the transformer could be loaded even more, thus decresing the unitilized lifetime over the loading period.

\section{PRoblem Formulation}

In this section the optimal multi-period DCOPF is formulated, where transformer thermal and aging dynamics are directly accounted for, along with transmission system losses.

\section{A. Base DCOPF with quadratic losses}

In the considered system the sets of buses, branches, conventional generators and windfarms are indicated with $\mathcal{N}, \mathcal{L}, \mathcal{G}$ and $\mathcal{W}$, respectively. While the transformers subsets with STR, DTR and $\mathrm{DTR}^{+}$are identified with $\mathcal{L}_{\mathrm{STR}}, \mathcal{L}_{\mathrm{DTR}}$ and $\mathcal{L}_{\mathrm{DTR}^{+}}$.

This study adopts a DC approximation of the full AC power flow equations. The active power flow on each branch is modelled by means of the Power Transfer Distribution Factor matrix $\boldsymbol{M}$. The matrix $\boldsymbol{M} \in \mathbb{R}^{|\mathcal{L}| \times|\mathcal{N}|}$ expresses the sensitivities of the power flow on each line with respect to the nodal power injections, where the $|\cdot|$ operator indicates the cardinality of the set. The power flow $f_{\ell}$ on branch $\ell$ can then be expressed as $f_{\ell}=\boldsymbol{M}_{\ell} \boldsymbol{P}^{\mathrm{inj}}$, where $\boldsymbol{M}_{\ell}$ is the $\ell$-th row of matrix $M$ and $P^{\text {inj }}$ represents a column vector of per unit power injection at each bus in the system.

Branch power losses $\boldsymbol{L}$ that are dissipated on transmission lines and transformers are expressed in terms of additional load demand $\boldsymbol{L}^{\text {bus }}$ at each bus. Losses that occur on branch $\ell$ are equally divided between sending and receiving bus by means of a loss allocation matrix $\boldsymbol{Y} \in \mathbb{R}^{|\mathcal{N}| \times|\mathcal{L}|}$ whose $(n, \ell)$ component is defined in (7).

$$
Y(n, \ell)= \begin{cases}0.5 & \text { if line } \ell \text { is connected to bus } n \\ 0 & \text { otherwise }\end{cases}
$$

The nodal power injection at bus $n$ can then be written as

$$
P_{n}^{\mathrm{inj}}=P_{g}+P_{w}-\left(P_{n}-P_{n}^{\mathrm{sh}}+L_{n}^{\mathrm{bus}}\right)
$$

where $P_{g}$ and $P_{w}$ represent thermal and wind power generation; $P_{n}$ and $P_{n}^{\text {sh }}$ represent net load demand and load shedding; $L_{n}^{\text {bus }}$ expresses nodal power losses where $L_{n}^{\text {bus }}=\boldsymbol{Y}_{n} \boldsymbol{L}$ and $\boldsymbol{Y}_{n}$ is the $n$-th row of the loss allocation matrix.

In order to consider quadratic power losses in the DCOPF while preserving its convexity, an iterative approach has been implemented. This method is inspired by existing algorithms in the literature that account for power losses by means of either linear or quadratic inequality constraints [9], [10]. However, as discussed in [11], these approaches may introduce additional fictitious losses in the presence of negative locational marginal prices due to congestions in the transmission system. Therefore, the iterative approach adopted in this study introduces an upper bound for power losses which is lowered accordingly at each iteration, should the losses be overestimated. The main steps are:

1) Set the upper bound for power losses to the value corresponding at the maximum power flow and set a tolerance $\delta$ for the convergence.

$$
L_{(1)}^{\max }=R f^{\max ^{2}}
$$

2) Solve DCOPF (12) and obtain resulting power flows $f_{(k)}$ and power losses $L_{(k)}$ for the $k$-th iteration.

3) Compute the difference between estimated losses and actual losses for the resulting power flows.

$$
\Delta L_{(k)}=L_{(k)}-R f_{(k)}^{2}
$$

If $\Delta L_{(k)} \leq \delta$ a solution is found, otherwise proceed to next step.

4) Update the upper bound for power losses with the losses corresponding to the power flows at step $k$, plus a small margin $\varepsilon$. Then return to step 2 .

$$
L_{(k+1)}^{\max }=R f_{(k)}^{2}+\varepsilon
$$

This iterative approach allows to solve the DCOPF with a quadratic representation of power losses while still preserving the original convexity, which guarantees uniqueness of the solution. This is achieved by gradually reducing the size of the feasible region for branch losses.

The optimization problem in (12) is the base multi-period DCOPF for a generic iteration $(k)$, where losses are accounted for. The objective is to find the optimal 24-hours day-ahead energy dispatch, which minimizes total generation cost over the period $\mathcal{T}$, where all constraints have to hold $\forall t \in \mathcal{T}$. The base lossy-DCOPF is formulated in a compact form in (12) and it is solved in the matlab-based modeling system CVX [12] using a Mosek academic license. 


$$
\min _{\Xi} \sum_{t \in \mathcal{T}}\left(\sum_{g \in \mathcal{G}} c_{g} P_{g, t}+\sum_{w \in \mathcal{W}} c_{w} P_{w, t}+\sum_{n \in \mathcal{N}} c^{\mathrm{sh}} P_{n, t}^{\mathrm{sh}}\right)
$$

$$
\begin{array}{ll}
\sum_{g \in \mathcal{G}}^{\text {s.t. }} P_{g, t}+\sum_{w \in \mathcal{W}} P_{w, t}-\sum_{n \in \mathcal{N}}\left(P_{n, t}-P_{n, t}^{\mathrm{sh}}+L_{n, t}^{\mathrm{bus}}\right)=0, \\
P_{g}^{\min } \leq P_{g, t} \leq P_{g}^{\max } & \forall g \in \mathcal{G}, \\
-\Delta P_{g}^{\max } \leq P_{g, t}-P_{g, t-1} \leq \Delta P_{g}^{\max } & \forall g \in \mathcal{G}, \\
-f^{\max } \leq f_{\ell, t} \leq f^{\max } & \forall \ell \in \mathcal{L}, \\
R f_{\ell, t}^{2} \leq L_{\ell, t} \leq L_{(k)}^{\max } & \forall \ell \in \mathcal{L}, \\
0 \leq P_{w, t} \leq P_{w}^{\mathrm{av}} & \forall w \in \mathcal{W}, \\
0 \leq P_{n, t}^{\mathrm{sh}} \leq P_{n} & \forall n \in \mathcal{N}
\end{array}
$$

where $\Xi=\left[P_{g, t}, P_{n, t}^{\mathrm{sh}}, P_{w, t}, L_{\ell, t}\right]$ is the set of decision variables that for each time step $t$ represent scheduled generator's output $P_{g, t}$, shed load $P_{n, t}^{\text {sh }}$ and dispatched wind power $P_{w, t}$ for every generator, bus and wind farm, respectively. Branch power losses $L_{\ell, t}$ are modelled by means of an auxiliary decision variable in conjunction with quadratic and linear inequality constraints.

The objective function in (12a) consists of three terms: the cost of dispatching conventional generators in the system over period $\mathcal{T}$ considering linear generation cost functions; a small, negligible cost for dispatching wind power in order to improve convergence of the algorithm; the additional cost of remedial corrective actions such as load shedding. Constraint (12b) enforces system day-ahead power balance for each hour in the considered time period. Constraints (12c) and (12d) impose operational limits on conventional generators in terms of their power outputs and ramping capabilities, whereas branch power flow are limited by constraints (12e). Branch power losses are bounded by constraints (12f). The lower bound consists in their correct quadratic representation, whereas the upper one is necessary to avoid the introduction of fictitious losses. This term is the sole to be iteratively reduced whenever power losses do not lie close enough to the lower boundary in terms of the chosen tolerance $\delta$. Lastly, constraints $(12 \mathrm{~g})$ and $(12 \mathrm{~h})$ impose physical limitations on the availability of wind power generation at each bus and the amount of load that can be shed, respectively.

\section{B. Additional constraints for STR}

In order to express the loading of the transformer on branch $\ell$, the power flow $f_{\ell}$ is scaled accordingly with the ratio of base system per unit power $S^{\text {base }}$ to the nameplate rating of the transformer $S_{\ell}^{\text {trf }}$. This scaling factor allows to show the loading $I_{\mathrm{trf}_{\ell}}$ defined in (13) relatively to the size of the transformer.

$$
I_{\mathrm{trr}_{\ell, t}}=f_{\ell, t} \frac{S^{\text {base }}}{S_{\ell}^{\mathrm{trf}}}
$$

The subset $\mathcal{L}_{\text {STR }}$ of transformers that are statically rated can then be represented in the base DCOPF (12) by introducing additional constraints (14) that limit the power flow on the corresponding branch $\ell$ for all considered time periods.

$$
-1 \leq I_{\mathrm{trf}_{\ell, t}} \leq 1, \quad \forall \ell \in \mathcal{L}_{\mathrm{STR}}, \quad \forall t \in \mathcal{T}
$$

\section{Additional constraints for DTR}

The loading of transformers that are dynamically rated is limited by operating hot-spot and top-oil temperatures rather than per unit load. Top-oil and hot-spot temperature variations are bounded by predefined values that ensure transformers are used within their thermal capabilities, according to state-ofthe-art loading guidelines. As discussed in [4], $\vartheta_{\text {top }}$ and $\vartheta_{\text {hst }}$ are modelled by means of quadratic inequality constraints which keep the resulting optimization problem a convex one. The extensive form of such values is provided in expressions (3) and (4) in Section II.

$$
\begin{array}{ll}
\vartheta_{\text {top }_{\ell, t}} \leq \vartheta_{\text {top }}^{\max } & \forall \ell \in \mathcal{L}_{\mathrm{DTR}}, \quad \forall t \in \mathcal{T} \\
\vartheta_{\text {hst } \ell, t} \leq \vartheta_{\mathrm{hst}}^{\max } & \forall \ell \in \mathcal{L}_{\mathrm{DTR}}, \quad \forall t \in \mathcal{T}
\end{array}
$$

Adding (15) and (16) to the base DCOPF formulation in (12) will consider the effect of having transformers dynamically rated during the 24-hours dispatch period.

\section{Additional constraints for DTR ${ }^{+}$}

The third loading strategy proposed in this paper takes into account not only temperature dynamics, but also transformer aging rate. This aspect is likely to play a role only in the long term, but it provides indication of how the transformers loading could be affected by cumulative lifetime consumption during continued high temperature operation. In order to do so, the exponential aging acceleration factor $\Lambda$ defined in Section III is included in the base DCOPF (12) by means of a set of linear inequality constraints that form a convex piece-wise linear approximation. Coefficients $m_{i}$ and $q_{i}$ in (17) are the slope and intercept values of the $i$-th tangent line that forms the approximation of $\Lambda$.

$$
\Delta \lambda_{t}=\max _{i}\left\{m_{i} \vartheta_{\mathrm{hst}_{t}}+q_{i}\right\} \approx \Lambda_{t}
$$

The expression in (17) relates the transformer hot-spot operating temperature $\vartheta_{\text {hst } t}$ to the corresponding incremental lifetime utilization $\Delta \lambda_{t}$. The cumulative lifetime utilization $\lambda_{t}$ is then evaluated in a discrete form in (18)

$$
\lambda_{t}=\lambda_{t-1}+\Delta \lambda_{t}
$$

As the transformer is dynamically rated considering lifetime consumption as well, constraints (15) and (16) are added to the base DCOPF (12) together with (19), where $\lambda_{0}$ represents the initial lifetime of the component, $\alpha$ the desired maximum aging rate which in this study has been assumed 1 and $t$ is the time counter during the simulation.

$$
\lambda_{\ell, t} \leq \lambda_{0}+\alpha t \quad \forall \ell \in \mathcal{L}_{\mathrm{DTR}^{+}}, \quad \forall t \in \mathcal{T}
$$

Ultimately, this approach allows to set a higher temperature limit on transformer operation as long as the designed lifetime consumption limit is not reached. Once the upper boundary of lifetime utilization is met, the binding constraint will switch 


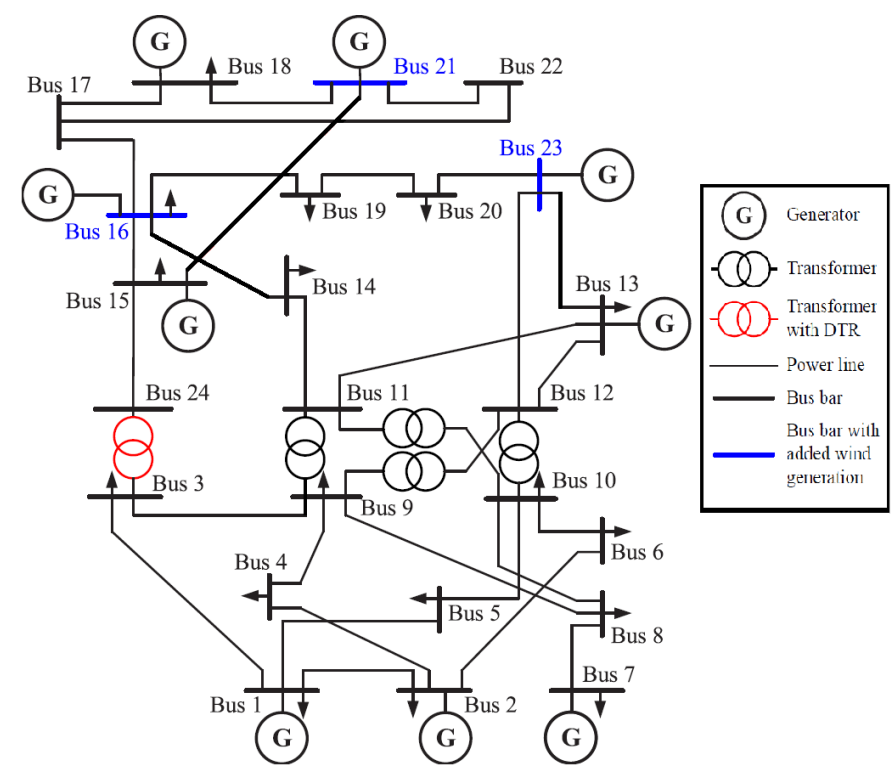

Fig. 2. Modified IEEE RTS 24-bus system [7]

from hot-spot temperature to used cumulative lifetime, thus limiting the operation of the component accordingly.

\section{CAse Study}

The IEEE RTS 24-bus network with additional wind generation from [7] has been adopted in this study with some modifications. Referring to Fig. 2, wind generation is concentrated at Bus 16, 21 and 23, whereas different nameplate ratings are considered for the transformer located between bus 3 and 24, namely $150 \mathrm{MVA}, 175 \mathrm{MVA}$ and $200 \mathrm{MVA}$. The data used to model transformer thermal dynamics is provided in [8].

The multi-period DCOPF problem of Section IV is solved in a moving window of 24 hours for the 3-year period between 2014 and 2016. Different test cases compare the system and transformer performance for STR, DTR with $\vartheta_{\text {hst }}^{\max }$ at $110^{\circ} \mathrm{C}$ and $\mathrm{DTR}^{+}$with $\vartheta_{\mathrm{hst}}^{\max }$ at $140^{\circ} \mathrm{C}$ combined with optimal lifetime utilization. The assumptions for the $\mathrm{DTR}^{+}$test case are quite conservative. The transformer is assumed to be in operation for 3 years until 2014 with varying HST resulting in cumulative loss-of-life of 1.5 years, which is cautiously chosen based on operational experience of large transformers.

Historical daily load profiles from the Danish power system have been scaled accordingly with respect to the peak demand in [7]. Total load demand is then increased by 25 percent during the central hours of each day in order to account for future network changes and to enhance the need for DTR in the given system. Historical wind power production data from Denmark have been used to simulated realistic wind power generation patterns as in [13], in order to consider application of DTR in a wind-dominated power system. Lastly, historical time series of ambient temperature from the same system have been used in the thermal rating algorithm for transformers. This allows to take the weather correlation between wind speed and ambient temperature into account. Such a correlation will be reflected between the available wind power generation and the loadability of transformers.

\section{RESULTS AND DISCUSSION}

The analysis starts from considering the overall impact of the tested loading strategies on the cumulative lifetime utilization of a 175 MVA transformer, shown in the left part of Fig. 3. It stands out as the STR approach practically does not cause any significant aging over the entire 3-years period, in agreement with conservative common loading practices. As opposed to STR, DTR ${ }^{+}$pushes the utilization of the component to the designed limit in less than 1 year. Once the maximum aging rate is met, the component will keep using the available designed lifetime at disposal, according to the proposed loading strategy presented in this paper.

The resulting aging profiles can be motivated by considering a three-day period in Fig. 4, where transformer loading and corresponding hot-spot temperatures are shown. As long as the transformer load is limited in terms of its per unit nameplate rating, the associated hot-spot temperature remains well below the allowed operational limits due to favourable weather conditions and dimensioning criteria. The resulting low HST profile coupled with the exponential aging acceleration factor shown in the right part of Fig. 3 will yield a close-to-zero aging rate for STR. However, in this condition the transformer branch constitutes a bottleneck in the grid, thus causing increased dispatch costs for the system.

Moving the transformer limiting factor from the per unit load to the hot-spot temperature by means of DTR allows to significantly increase the power flow. This would help releasing grid congestions and dispatching more wind power generation from the buses where it is located, at a cost of increasing the lifetime utilization of the component. This mechanism is further enhanced by considering $\mathrm{DTR}^{+}$, which allows the transformer hot spot temperature to be set even higher, as long as the aging rate does not reach the predefined limit, as shown in Fig. 3. Once the maximum aging rate is reached, the constraint on lifetime utilization will prevent the hot-spot temperature to reach the maximum value, thus resulting in a lowered loading capability.
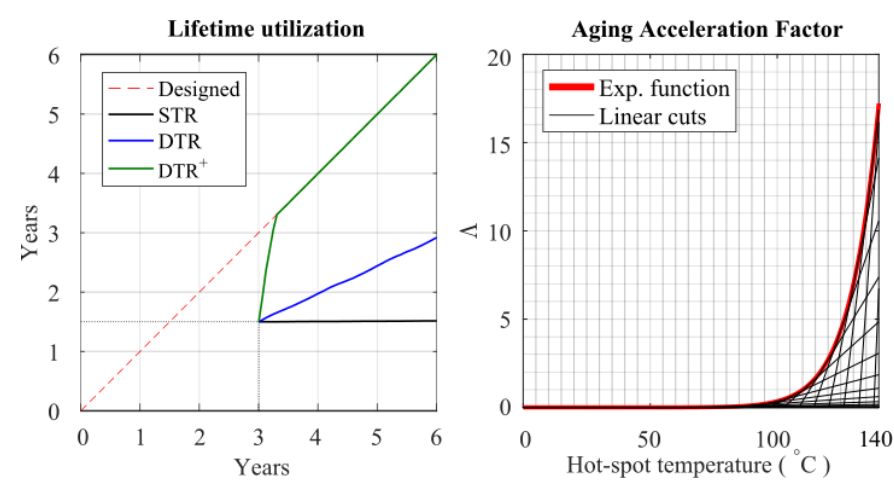

Fig. 3. Cumulative lifetime utilization over the 3-years period for different loading strategies (left). Exponential aging acceleration factor and fitted linear approximations (right). 

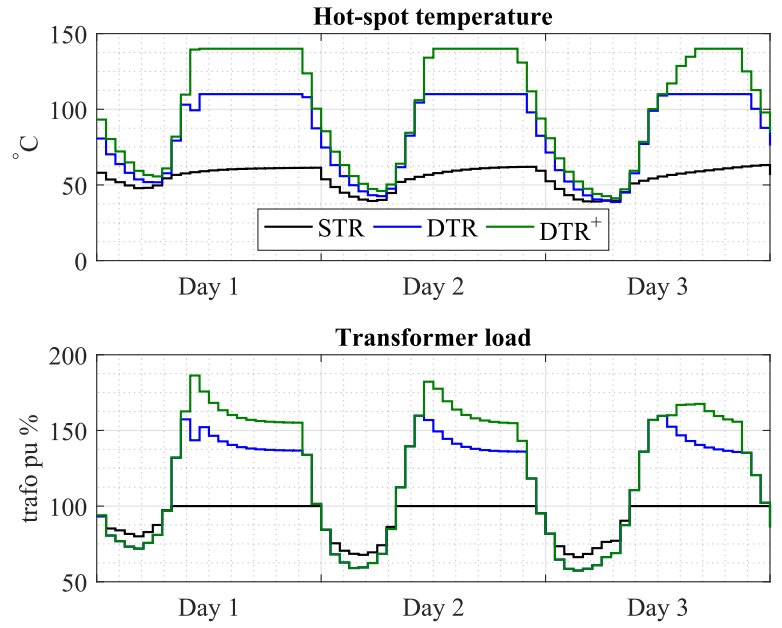

Fig. 4. Transformer hot-spot temperature (above) and transformer load (below). Focus on three days for STR, DTR and DTR ${ }^{+}$

The operation of the transformer beyond its nameplate rating causes additional power losses, not only in the component that is being dynamically rated, but in the remainder of the system as well. This aspect can be seen in Fig. 5, where transformer and system losses are shown for the same 3days period. Relieving grid congestions by means of DTR or $\mathrm{DTR}^{+}$will result in increased power flows across the grid, which will in turn cause additional system losses. Despite increasing system losses, the solution of the multi-period DCOPF suggests that there would still be economic benefits from the increased power flow in the grid as summarized in Table II for different sizes of transformers.

TABLE II

DISPATCH COST DECREASE \& LIFETIME UTILIZATION COMPARED TO STR AFTER 1 YEAR

\begin{tabular}{ccccc}
\hline $\begin{array}{c}\text { Transformer Size } \\
\text { (MVA) }\end{array}$ & \multicolumn{2}{c}{$\begin{array}{c}\text { Cost Reduction } \\
(\boldsymbol{\%})\end{array}$} & \multicolumn{2}{c}{$\begin{array}{c}\text { Used Lifetime } \\
(\boldsymbol{\%})\end{array}$} \\
\hline & DTR & DTR $^{+}$ & DTR & DTR $^{+}$ \\
\cline { 2 - 5 } & -10.1 & -11.5 & +41.5 & +166 \\
$\mathbf{1 5 0}$ & -8.1 & -8.8 & +31.1 & +166 \\
$\mathbf{1 7 5}$ & -6.4 & -7.1 & +24.2 & +166 \\
\hline
\end{tabular}

\section{CONCLUSION}

This paper proposes a novel approach for optimal transformer utilization in the day-ahead energy dispatch. This approach incorporates both thermal and aging dynamics into a convex optimization problem based on a multi-period DCOPF, while accounting for quadratic power losses in the system. The proposed algorithm allows transformer utilization to be maximized ensuring that neither thermal nor aging rate limits are violated during operation. The IEEE RTS 24-bus network with additional wind generation has been used as a test case for daily load dispatch over a period of 3 years. The results suggest that the proposed DTR algorithm reduces the cost of load dispatch and yields a substantial increase in
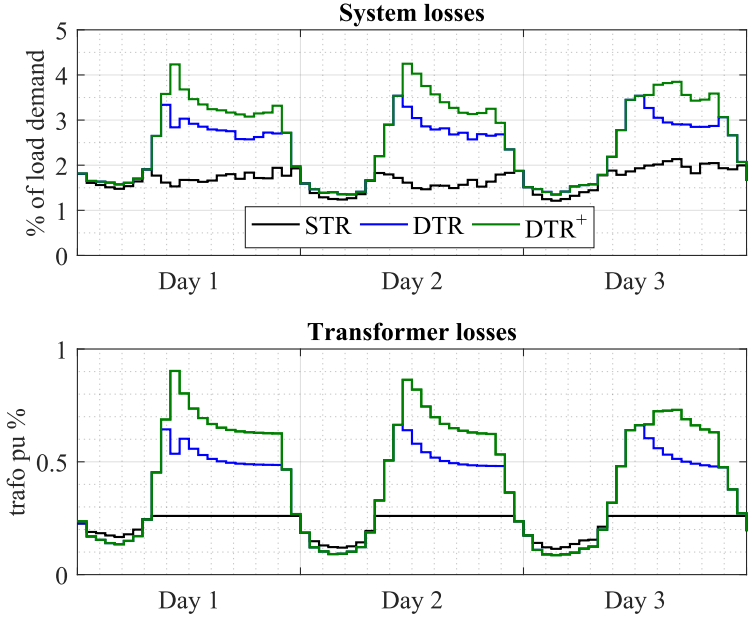

Fig. 5. Total system losses (above) and transformer losses (below). Focus on three days for STR, DTR and DTR ${ }^{+}$

network capacity. It is also observed that accounting for the temperature-dependent aging rate can allow a better utilization of the transformer designed lifetime. This aspect is likely to improve the business case for applications with limited asset lifetime like offshore windfarms. It could also be beneficial for TSO which have to face rapid growth of renewable-based generation and postpone the required grid reinforcements.

\section{REFERENCES}

[1] J. Rossenlind, "Lifetime modeling and management of transformers," 2013.

[2] "IEEE guide for loading mineral-oil-immersed transformers and stepvoltage regulators - redline," IEEE Std C57.91-2011 (Revision of IEEE Std C57.91-1995) - Redline, pp. 1-172, Mar 2011.

[3] IEC, "IEC 60076-7:2005 power transformers - part 7: Loading guide for oil-immersed power transformers," vol. 60076, no. 7, 2005.

[4] N. Viafora, K. Morozovska, and S. H. Kazmi, "Day-ahead Dispatch Optimization with Dynamic Thermal Rating of Transformers and Overhead Lines," EPSR Special Issue: Increasing transmission capacity of overhead lines to improve RES integration, 2018.

[5] M. Humayun, M. Degefa, A. Safdarian, and M. Lehtonen, "Utilization improvement of transformers using demand response," IEEE transactions on power delivery, vol. 30, no. 1, pp. 202-210, Feb 2015.

[6] G. B. et al., "Optimization of transformer loading based on hot-spot temperature using a predictive health model," in Proceedings of the 2010 International Conference on Condition Monitoring and Diagnosis (CMD 2010), Tokyo, Japan. IEEE, 2010, pp. 914-917.

[7] C. Ordoudis, P. Pinson, and M. Zugno, "An updated version of the IEEE rts 24-bus system for electricity market and power system operation studies," Technical University of Denmark (DTU), pp. pp. 1-5, 2016.

[8] D. Susa, M. Lehtonen, and H. Nordman, "Dynamic thermal modelling of power transformers," IEEE transactions on Power Delivery, vol. 20, no. 1, pp. 197-204, 2005.

[9] R. A. Jabr, "Modeling network losses using quadratic cones," IEEE Transactions on Power Systems, vol. 20, no. 1, pp. 505-506, Feb 2005.

[10] T. N. dos Santos and A. L. Diniz, "A dynamic piecewise linear model for dc transmission losses in optimal scheduling problems," IEEE Transactions on Power Systems, vol. 26, no. 2, pp. 508-519, May 2011.

[11] O. W. Akinbode and K. W. Hedman, "Fictitious losses in the dcopf with a piecewise linear approximation of losses," in 2013 IEEE Power Energy Society General Meeting, July 2013, pp. 1-5.

[12] M. Grant and S. Boyd, "CVX: Matlab software for disciplined convex programming, version 2.1," http://cvxr.com/cvx, Mar. 2014.

[13] N. Viafora, S. Delikaraoglou, P. Pinson, and J. Holbøll, "Chanceconstrained optimal power flow with non-parametric probability distributions of dynamic line ratings," in MATPOWER, Nov 2018, pp. 1-6. 